\title{
COVID-19 Vaccination and Mental Health Distress
}

$J$ Gen Intern Med 37(4):1020-1

DOI: $10.1007 / \mathrm{s} 11606-021-07226-1$

(c) Society of General Internal Medicine 2021

In addition to its grave physical health impact and associated mortality, the COVID-19 pandemic had a significant negative impact on mental health of affected populations. ${ }^{1,2}$ With widespread vaccination, there are now hopes that the pandemic can be finally brought under control. While the impact of vaccination on the incidence of COVID-19 infection and related physical health consequences are actively investigated, with rare exceptions, ${ }^{3}$ the mental health impact of vaccination remains unexplored. This report examines association of COVID-19 vaccination with significant psychological distress in a large-scale survey of the US population conducted in the first half of 2021 - a period of widespread implementation of COVID-19 vaccination.

\section{METHODS}

Data from the Census Bureau's Household Pulse Survey (HPS), ${ }^{4}$ a nationally representative online cross-sectional survey of adults conducted regularly since April 2020, were analyzed. The HPS is designed to provide near real-time information on household experiences during the pandemic. Potential participants were selected from the Census Bureau's Master Address File. Selected adults were invited to participate by email and text messages. The sample sizes in each state were determined based on an anticipated response rate of $5 \%$.

Data for this report were limited to HPS waves 22 to 33, conducted between January 6 and July 5, 2021. Each wave interviewed approximately 70,000 (range $=66,262-80,567)$ adults aged $\geq 18$. Questions about COVID-19 vaccination were first introduced in HPS wave 22.

Along with questions about socio-demographic characteristics, respondents completed versions of the Generalized Anxiety Disorder (GAD-2) and the Patient Health Questionnaire (PHQ-2) to screen for significant psychological distress over the past 1-week period based on a score of $\geq 3$. $^{2}$

Received: 29 August 2021

Accepted: 19 October 2021

Published online January 19, 2022
This study analyzed the association of receipt of COVID19 vaccination with GAD-2 and PHQ-2 using logistic regression models, adjusting for age, sex, race/ethnicity, education, marital status, employment, household composition, household income, health insurance, and assessment period. In addition, analyses adjusted for COVID-19 test-positive rates and proportion of adults vaccinated, both for each state and in each period, using data from Centers for Disease Control. ${ }^{5}$

In further analyses, variations in the strength of association of vaccination with GAD-2- and PHQ-2-positive screens across time periods were examined by testing the interaction of time with vaccination in the regression models.

Analyses were adjusted using doubly robust inverse probability of treatment weighing (IPTW) using propensity scores. ${ }^{6}$ Acceptable balance of covariates after weighting was checked and confirmed. Additionally, sampling weights and replicate weights were included in the analyses to adjust for survey non-response and to provide nationally representative estimates. Analyses were conducted using Stata 17 software. All percentages are weighted.

\section{RESULTS}

A total of 884,697 adults participated in the 12 surveys. Of these, 878,978 (99.3\%) responded to questions about COVID-19 vaccination; 506,369 (50.7\%) reported having received a vaccine. Fully $22.3 \%(n=188,780)$ of the interviewed adults screened positive on GAD-2 and $18.8 \%$ $(n=144,764)$ on PHQ-2.

Those who had received COVID-19 vaccine were less likely than the un-vaccinated to screen positive on GAD-2 (28.6\% vs. $30.7 \%$, respectively, adjusted odds ratio $[\mathrm{AOR}]=0.90,95 \%$ confidence interval $[\mathrm{CI}]=0.86-0.93$, $p<0.001)$ and on PHQ-2 (22.8\% vs. $25.3 \%, \mathrm{AOR}=0.86$, $95 \% \mathrm{CI}=0.83-0.90), p<0.001)$ across the years. However, the association of vaccination status with both GAD-2 and PHQ-2 changed with passage of time as indicated by significant interaction terms in regression models (GAD2: $\mathrm{AOR}=1.01,95 \% \mathrm{CI}=1.005-1.2, p=0.002$; PHQ-2; $\mathrm{AOR}=1.2,95 \% \mathrm{CI}=1.1-1.3, p<0.001)$ (Figs. 1 and 2). 


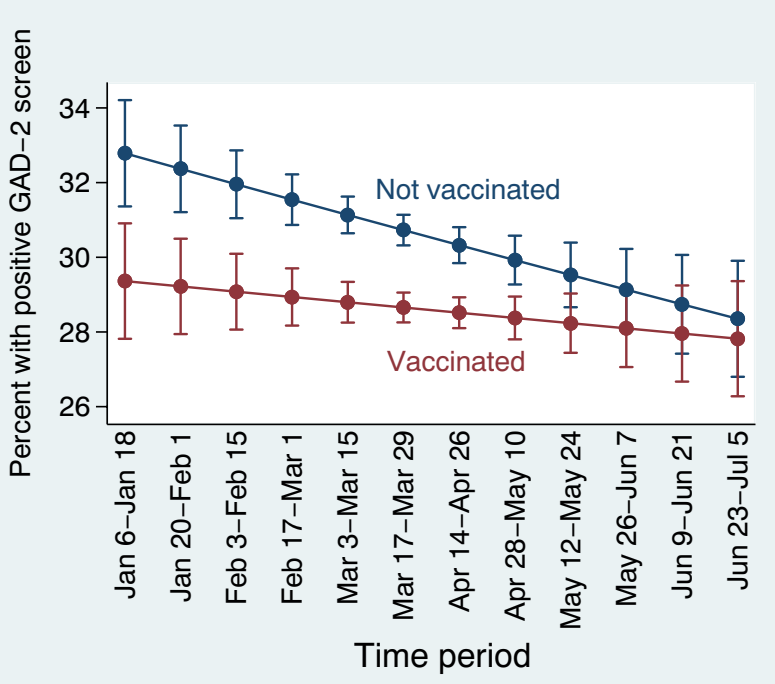

Fig. 1 Association of COVID-19 vaccination with screening positive on the Generalized Anxiety Disorder-2 item questionnaire (GAD-2 $\geq 3$ ) over the January 6 to July 5, 2021, period in the US Household Pulse Survey

\section{DISCUSSION}

This study found small but significant associations between COVID-19 vaccination and decline in psychological distress among adults in the first part of 2021. Extrapolating to the US adult population, these estimates correspond to over 5-6 million fewer GAD-2- and PHQ-2 screen-positive adults.

The association of vaccination with psychological distress was more pronounced in the early phase of vaccine roll-out. This effect cannot be attributed to the earlier vaccination of older adults because the analyses adjusted for age.

While a causal connection cannot be drawn from these cross-sectional data, the findings are consistent with a recent report that identified reduction in psychological distress following vaccination, likely mediated by reduction in fears related to COVID-19 infection and its consequences. ${ }^{3}$ Better understanding of factors that contribute to mental health distress and its alleviation during the COVID-19 pandemic may help in predicting the mental health impact of future epidemics and other mass disasters.

\section{Ramin Mojtabai, $M D, P h D, M P H^{1}$}

${ }^{1}$ Department of Mental Health, Bloomberg School of Public Health and Department of Psychiatry, School of Medicine, Johns Hopkins University, 624 North Broadway, Room 797, Baltimore, MD 21205, USA

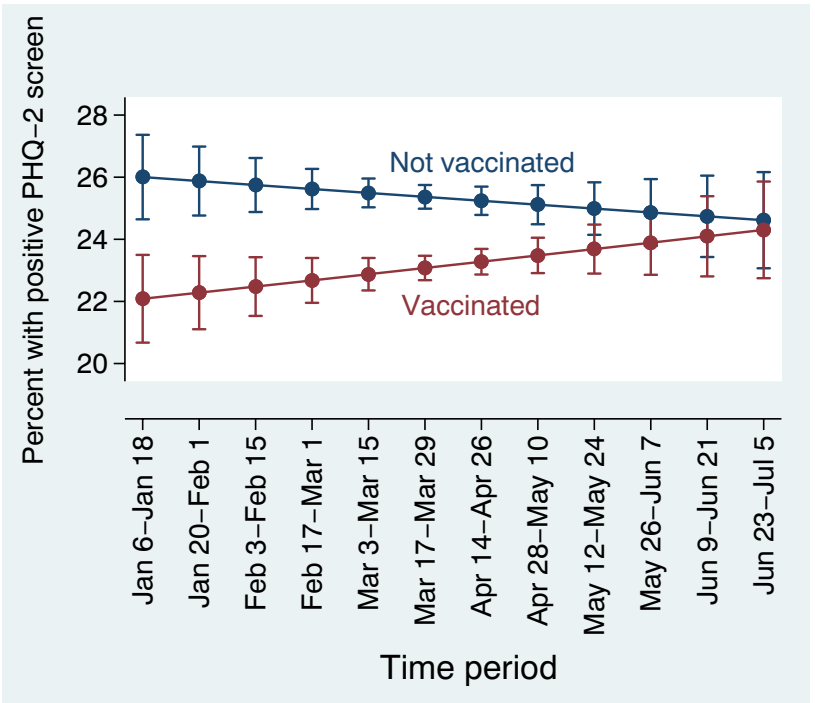

Fig. 2 Association of COVID-19 vaccination with screening positive on the Patient Health Questionnaire-2 item (PHQ-2 $\geq 3$ ) over the January 6 to July 5, 2021, period in the US Household Pulse Survey

Corresponding Author: Ramin Mojtabai, $\mathrm{MD}, \mathrm{PhD}, \mathrm{MPH}$; Department of Mental Health, Bloomberg School of Public Health and Department of Psychiatry, School of Medicine, Johns Hopkins University, 624 North Broadway, Room 797, Baltimore, MD, 21205, USA (e-mail: rmojtab1@jhu.edu).

\section{Declarations}

Conflict of Interest The author declares no conflict of interest.

\section{REFERENCES}

1. 1.Cai C, Woolhandler S, Himmelstein DU, Gaffney A. Trends in anxiety and sepression symptoms suring the COVID-19 pandemic: Results from the US Census Bureau's Household Pulse Survey. J Gen Intern Med. 2021;36(6):1841-1843.

2. 2.Berkowitz SA, Basu S. Unemployment insurance, health-related social needs, health care access, and mental health during the COVID-19 pandemic. JAMA Intern Med. 202 1;181(5):699-702.

3. Koltai J, Raifman J, Bor J, McKee M, Stuckler D. Does COVID19 vaccination improve mental health? A difference-in-difference analysis of the Understanding Coronavirus in America study. medRxiv. 2021.

4. United States Census Bureau. Household Pulse Survey Public Use File (PUF). 2021; Available at: https://www.census.gov/progr ams-surveys/household-pulse-survey/datasets.html. Accessed August 18, 2021.

5. Centers for Disease Control and Prevention. COVID Data Tracker. 2021; Available at: https://covid.cdc.gov/covid-data-tracker/\# datatracker-home. Accessed August 18, 2021.

6. 6.Funk MJ, Westreich D, Wiesen C, Sturmer T, Brookhart MA, Davidian M. Doubly robust estimation of causal effects. Am J Epidemiol. 2011;173(7):761-767.

Publisher's note Springer Nature remains neutral with regard to jurisdictional claims in published maps and institutional affiliations. 